\title{
New dental hygiene protocol for systemic disease prevention
}

\begin{abstract}
Malpositioned teeth are prone to greater accumulations of bacterial plaque due to the difficulty of proper dental hygiene and are subject to a unique pathogenic bacteria microflora which has the potential to increase the severity of gingival inflammation and accelerate the progression of periodontal disease. Furthermore, specific gram-negative bacteria in this microbial floral have been implicated in a variety of systemic diseases largely by the circulatory dissemination of inflammatory cytokines. Orthodontic treatment by way of conservative and nonsurgical methods or by the facilitation of natural eruption or Orthoeruption $^{\mathrm{TM}}$ of these teeth is protective for these disease-causing bacteria. Orthodontic treatment planning is therefore a valuable therapeutic periodontal option that should be considered with mainstream periodontal treatment planning. A new dental hygiene protocol is proposed which integrates immediate application of orthodontic brackets and Orthoeruption $^{\mathrm{TM}}$ of teeth. This new protocol simultaneously provides multiple patient benefits in a relatively short period of time.
\end{abstract}

Keywords: orthodontics, orthoeruption, orthodontosis, orthodontitis, gram- negative oral bacteria, periodontal disease, medicine, protocol, dental hygiene
Volume 10 Issue 5 - 2019

\author{
Anthony D Viazis,' Tom C Pagonis ${ }^{2}$ \\ Orthodontist, Private Practice, Dallas, Texas, USA \\ ${ }^{2}$ Associate Clinical Professor, Tufts University, School of Dental \\ Medicine, Former Faculty, Harvard School of Dental Medicine, \\ Boston, MA, USA
}

Correspondence: Tom C Pagonis, Associate Clinical Professor, Tufts University School of Dental Medicine; former faculty, Harvard School of Dental Medicine, Boston, MA, USA, Email dental.research@comcast.net

Received: October 03, 2019 | Published: October 09, 2019

\section{Introduction}

The convergence of orthodontic and periodontal considerations in diagnosis and treatment planning should become common place in clinical practice. While there has been a large number of systemic conditions ${ }^{1}$ and specific periodontal pathogens linked to periodontitis, the bacterial flora of malpositioned teeth present a unique challenge both in the pathophysiology of periodontal disease and possible systemic consequences. The pathophysiology of periodontal disease is modulated by the host immune response and tissue structural considerations, but the key components in disease are associated with the bacterial flora in plaque biofilm. ${ }^{1}$ Periodontal pathogens produce localized damage in two different ways:

\section{Directly through tissue necrosis and apoptosis}

2. Indirectly through damage via the host response. ${ }^{2,3}$

It is important to note that gram-negative anaerobic bacteria including Porphyromonas gingivalis, Eikenella corrodens and Treponema denticola are most responsible for causing local tissue damage. This phenomenon illustrates the nature of chronic periodontitis as a polymicrobial disease resulting from the overgrowth of a limited number of bacterial species causing tissue destruction. ${ }^{4}$ The direct mechanism of periodontal tissue destruction for this profile of bacteria includes damage to crevicular epithelium, leukocyte impairment, degradation of immunoglobulins, degradation of fibrin, collagen and the activation of complement and bone resorption with the release of lipopolysaccharide (LPS) or endotoxin. ${ }^{5-7}$ For malpositioned teeth, the disease process is magnified and understanding the interrelation and impact of periodontal disease and orthodontic treatment is particularly important.

The architecture of malpositioned teeth facilitate the accumulation of bacterial plaque by creating physical barriers to self-cleaning which directly contributes to gingival inflammation..$^{8-10}$ The literature also confirms that the boney and soft tissue architecture created by malpositioned teeth contributes to the creation of a unique bacterial flora. In 2000, Chung, Vanarsdall, and co-workers ${ }^{11}$ concluded that malpositioned anterior dentition in adults exhibited greater plaque accumulation, a greater number of periodontopathogens present in subgingival plaque with a significantly more common presence of Fusobacterium species, Capnocytophaga species, $B$ rectus and $P$ micros when compared with non-malpositioned teeth. In addition, a study by Thornberg and co-workers ${ }^{12}$ examined levels of eight periodontal pathogens; Actinobacillus actinomycetemcomitans, Eikenella corrodens, Fusobacterium nucleatum, Porphyromonas gingivalis, Prevotella intermedia, Tannerella forsythia, Treponema denticola, and Campylobacter rectus on adolescents pre-operatively, peri-operatively and post-operatively. They concluded that orthodontic treatment had a positive effect post-treatment and protective for four of the pathogens, namely Eikenella corrodens, Fusobacterium nucleatum, Treponema denticola, and Campylobacter rectus. Also of note is that the severity of crowding and malocclusion, if left untreated, increases with age with a clinically notable increased severity of periodontal problems.

The purpose of this paper is three-fold:

i) To expand on the microbiology of specific periodontal pathogens of malpositioned teeth and their possible systemic manifestations,

ii) To emphasize that orthodontic treatment of malpostioned teeth is no longer an elective procedure but rather a routine therapeutic treatment modality.

iii) To introduce a new dental hygiene protocol which incorporates immediate application of brackets for Orthoeruption ${ }^{\mathrm{TM}}$ of teeth as a therapeutic adjunct. 


\section{Methodology}

The following pathogens have been identified as significant players in the bacterial microbial flora of malpositioned teeth. The facilitation of natural eruption or Orthoeruption ${ }^{\mathrm{TM}}$ of malpositioned teeth serves both to significantly decrease bacteria and provides a protective mechanism against these bacteria.

Eikenella corrodens is a pleomorphic, fastidious facultative anaerobic gram negative bacillus that can grow in both aerobic and anaerobic conditions. It is considered endogenous to the mouth and upper respiratory tract often recovered as a constituent of polymicrobial infections most commonly associated with streptococci. It is identified as indolent, a characteristic of infection which generally takes at least one week from tissue invasion to clinical manifestation. The head and neck are the most common sites of infection for both children and adults which includes pneumonia, septic emboli and other respiratory tract infections. Eikenella has also been associated with infection in insulin-dependent diabetic patients. This pathogen has been reported in gynecologic infections, human bite infections and has been recovered from synovial fluid, bone, cerebrospinal fluid, the brain and from subdural visceral infections. It is one of the HACK group (i.e. Haemophilus parainfluenzae, Aggregatibacter actinomycetemcomitans, Cardiobacterium hominis, Eikenella corrodens, Kingella kingae) of infections which are a group of fastidious gram-negative bacteria causing infective endocarditis (IE). These organisms can travel to distant organ sites and cause disease. It is postulated they enter the blood stream during dental procedures (i.e. dental cleaning) or as a consequence of periodontal disease. ${ }^{13}$ The clinical manifestation of IE from E. corrodens is usually associated with poor oral hygiene.

Treponema denticola is a gram-negative anaerobic motile spirochete considered a key periodontal microbial flora constituent associated both with the incidence and severity of periodontal disease. Along with Prophymonas gingivalis and Tannerella forsythia it forms the red-complex of bacteria and is linked to severe manifestations in immunocompromised patients. Treponema denticola, is implicated in a wide variety of systemic conditions from heart disease to low birth weights. Several studies have associated the dissemination of Treponema denticola in periodontal disease to the heart ${ }^{14}$ in cases of coronary disease. This pathogen has also been detected in atherosclerotic plaques of cardiac vessel,${ }^{15}$ and in sites of esophageal cancer. $^{16}$

One of the most significant discoveries regarding Treponema denticola is in its strong correlation to Alzheimer's disease (AD), a neurodegenerative disease associated with the geriatric population with an incidence that increases significantly with age. ${ }^{17}$ The connection or affiliation between periodontal disease and Alzheimer's disease appears to be related to increased levels of proinflammatory cytokines which disseminate systemically and an accompanying elevated C-reactive protein level. There is then a tendency to induce further inflammation by way of forming distinct compounds such as Abeta-amyloid 1-42 which in turn stimulate microglial cells. While microglial cells are neuroprotective for younger individuals their function is compromised in older, vulnerable populations resulting in the production of neurotoxic substances when they are exposed to systemic inflammation. ${ }^{18-20}$

Fusobacterium nucleatum, is also gram-negative anaerobic pathogen that is a quite common oral commensal bacterium and a key periodontal pathogen. These is a significant amount of literature that periodontal disease caused by Fusobacterium nucleatum, is also associated with a wide spectrum of human diseases.
Periodontal disease caused by $F$. nucleatum has been associated with preterm births with cells that have been isolated from amniotic fluid and the placenta. It is now accepted that these and other findings provide evidence for a causal relationship between periodontal disease caused by F. nucleatum and some preterm births. Additional theories of systemic action include possible uterine tissue invasion and infection..$^{21,22}$

This bacterium is highly adhesive and researchers postulate that this allows for horizontal gene transfer from co-exiting bacteria. $F$. nucleatum is highly associated with colorectal cancer which ranks as the third most prevalent form of cancer and fourth most common cause of cancer death in the world. ${ }^{23}$ Evidence now shows that levels of $F$. nucleatum are significantly elevated in patients with colorectal cancer and this pathogen is present in up to one third of patients with colorectal cancer. These high levels of $F$ nucleatum are particularly harmful as they are capable of activating signaling pathways in tumorigenesis while blocking human immune cell activity to fight tumor formation. In effect the high levels of $F$. nucleatum simultaneously promotes tumor formation while inhibiting human T-cell formation needed to suppress tumor growth. ${ }^{24,25}$ This pathogen is also implicated in appendicitis, inflammatory bowel disease and liver abscess. ${ }^{26}$

Campylobacter rectus, is a gram-negative, microaerophilic, motile bacterium, which is present in plaque and plays a significant role in the pathogenesis of periodontal disease..$^{27}$ The bacterium's virulence is associated with its surface anatomy including the flagellum and surface protein layer (S-layer). Studies have identified that Campylobacter rectus has the ability to translocate in vivo from a distant site of infection to human fetal tissue thus implicated in adverse pregnancy outcomes including preterm delivery. ${ }^{28}$

\section{Discussion}

Based upon the definitions of Orthodontitis ${ }^{\mathrm{TM}}$, orthodontic treatment even for the periodontally compromised patient should be directed towards mimicking and continuing the light forces of natural eruption thereby stimulating bone remodeling around displaced roots. This eliminates the need for extraction therapy and in the case of a periodontally involved patient, it stimulates favorable bone remodeling. Furthermore, this mechanically assisted continuation of the eruption has been defined as Orthoeruption ${ }^{\mathrm{TM}}{ }^{29}$ and allows for the uprighting of displaced roots into a straight position as if the teeth erupted in that position. Much like the remodeling of alveolar bone to accommodate teeth during the natural eruption, orthodontically induced eruption or Orthoeruption ${ }^{\mathrm{TM}}$ stimulates the continued remodeling of alveolar bone to accommodate the roots towards their final naturally erupted position. Fastbraces Technologies ${ }^{\circledR}$ patented methods now make it possible to Orthoerupt ${ }^{\mathrm{TM}}$ or initially align malpositioned roots typically in 60 to 90 days. This provides multiple benefits which include alveolar bone remodeling and the restoration of the dental arch to its appropriate natural size and shape. In addition, it eliminates the unique bacterial microbial flora associated with malpositioned teeth and creates the proper alignment needed to facilitate both professional and personal dental hygiene. The relatively short treatment time with accompanying benefits makes this an attractive and acceptable treatment option for patients.

The specific pathogens discussed as unique to the microflora of malpositioned teeth does not imply that they are the only pathogenic bacteria that have systemic disease consequences. Indeed, periodontal infections are polymicrobial in origin and the microflora of malpositioned are populated by and affected by many periodontal pathogens that are linked to systemic disease (Figure 1). In 2014 the authors identified the above referenced pathogens 
and introduced a disease-based or biologically verifiable model of orthodontic diagnosis based on alveolar bone clinical morphology rather than subjective arbitrary "ideals" of the Angle Classification of Malocclusion..$^{29}$ The disease-based term Orthodontosis ${ }^{\mathrm{TM}}$ was introduced and defined as the non-inflammatory deficiency of the alveolar bone in the axial plane caused by the displaced $\operatorname{root}(\mathrm{s})$ of the tooth, typically palatally or lingually. We further hypothesized that Orthodontosis ${ }^{\mathrm{TM}}$ is the underlying precursor to the clinical manifestation of a cleft or, as defined, a split or separation of bone in craniofacial development. The soft tissue consequence or resulting excess soft tissue and chronic inflammation leads to the diagnostic term of Orthodontitis ${ }^{\mathrm{TM}}$. These terms serve as the basis of the cause or illness and disease in orthodontics. In 2017 the authors created the first set of classification terms which capture our disease-based model and identify Alveolar Hypoplasia and Alveolar Hyperplasia in both the maxilla and mandible. ${ }^{30}$ This subsequently led to our 2018 paper which introduced and proposed the The Viazis Classification of Malocclusion $^{31}$ as the new system for orthodontic classification which is based on alveolar bone morphology. Based upon this increased convergence of orthodontics and periodontal disease particularly when evaluating alveolar bone morphology, the unique and pathogenic bacterial microflora of malpositioned teeth and even the possible systemic manifestations of periodontal disease, the authors further proposed the diagnostic term Orthodontitis ${ }^{\mathrm{TM}}$ as the distinct gingivitis around malpositioned teeth. ${ }^{32}$ It is now time to further translate this knowledge into clinical treatment planning and practice.

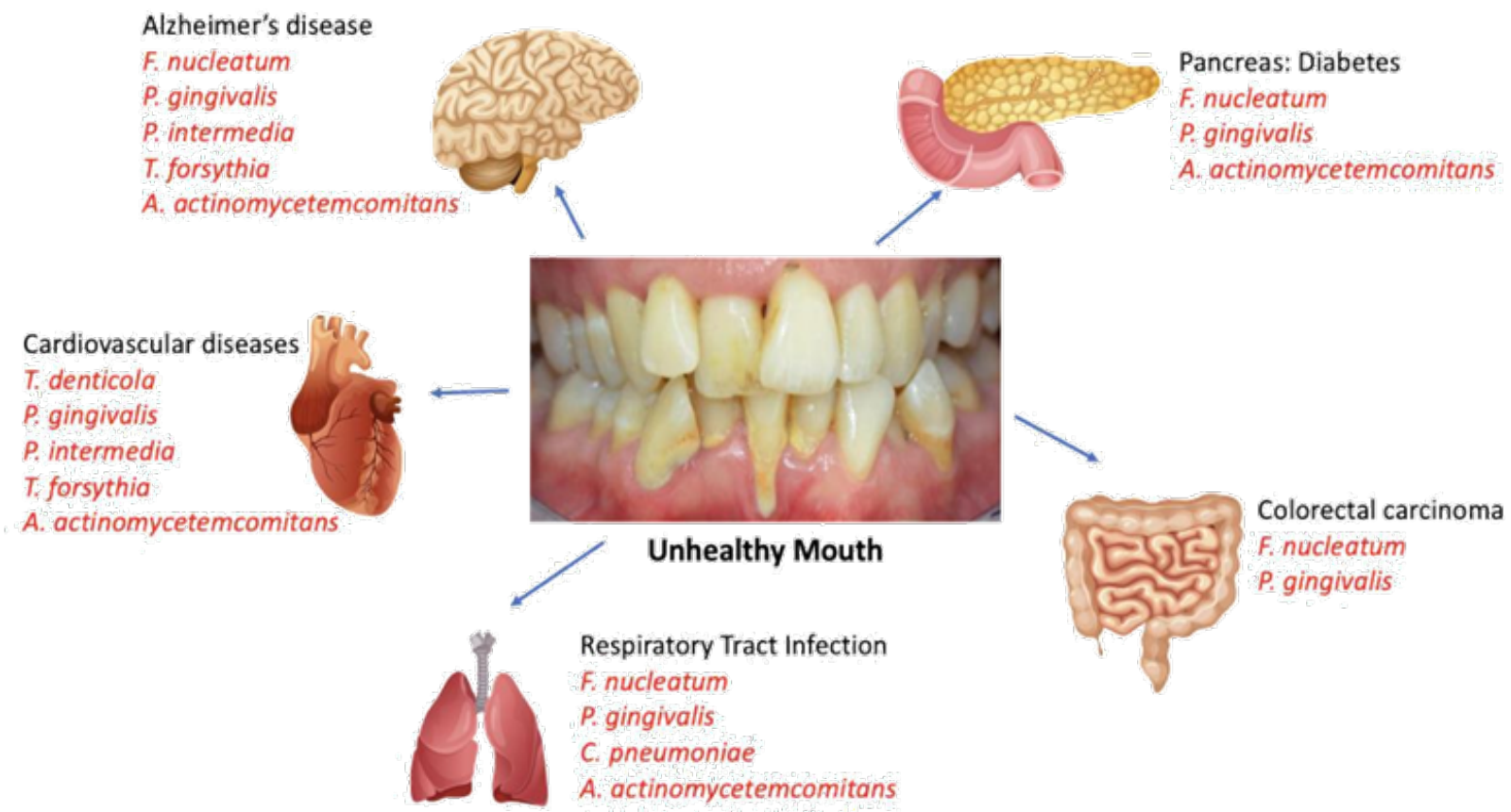

Figure I Organ specific systemic manifestations from periodontal pathogens of an unhealthy mouth.

The periodontal - systemic continuum has been well documented in the literature and is gaining greater acceptance when studying specific pathogens and accompanying systemic conditions. Much emphasis has been made to aggressively treat periodontal disease on a local level both for oral and systemic health. Yet, there is a substantial void in periodontal treatment planning by way of facilitating natural eruption or Orthoeruption ${ }^{\mathrm{TM}}$ of malpositioned teeth. By definition natural eruption is root movement which is followed by alveolar bone growth. The new bone around the final position of the naturally erupted root demonstrates the alveolar bone growth that occurred during eruption. If not, the root would find itself outside the alveolar bone housing. The strong connection between alveolar bone maintenance and its ability to remodel is evident both from the alveolar bone lacunae and canuliculi ultrastructure which optimizes nutrient availability with ample blood flow through the periodontal ligament and by the catabolic effect that results in bone resorption following tooth extraction. So a de facto consequence of root movement in natural eruption is the alveolar bone growth around the new position. Much like the remodeling of alveolar bone to accommodate teeth during natural eruption, orthodontically induced eruption or Orthoeruption ${ }^{\mathrm{TM}}$ may stimulate the continued remodeling of alveolar bone to accommodate the roots towards their final naturally erupted position. ${ }^{29-32}$

Whether malpositioned teeth appear periodontally healthy in younger patients or whether they are periodontally compromised in older patients, orthodontic treatment is required for preventive or protective purposes in younger patients and for therapeutic purposes in older patients. As referenced in this paper and in our previous publications, ${ }^{29,32}$ malpositioned teeth have a unique microbial flora which is characterized by highly pathogenic bacteria. If left untreated local consequences of periodontal disease are magnified. It is logical to infer that if orthodontic treatment is protective for these bacteria and if there are systemic manifestations in disease for these pathogens, it follows to advocate therapeutic orthodontic treatment for periodontal 
and systemic purposes. The authors propose a contemporary protocol that integrates Orthoeruption ${ }^{\mathrm{TM}}$ as a mechanism to facilitate both professional and home cleaning while creating a more favorable periodontal microflora. This protocol (Figure 2) consists of

1. Diagnosis: record which teeth have Orthodontitis ${ }^{\mathrm{TM}}$ and which have Gingivitis.

2. Thoroughly clean labially positioned teeth (gingivitis) and use best efforts to clean lingually inclined teeth (Orthodontistis ${ }^{\mathrm{TM}}$ ).

3. Start Orthoeruption ${ }^{\mathrm{TM}}$ with immediate application of brackets on anterior teeth diagnosed with Orthodontitis ${ }^{\mathrm{TM}}$ which are typically the lateral incisors.

4. At a subsequent appointment apply brackets on remaining teeth diagnosed with Orthodontitis ${ }^{\mathrm{TM}}$ along with the application of brackets on molars for anchorage. This new protocol is both efficient and effective because the initial application of braces takes place at the initial dental hygiene appointment.

\section{Conclusion}

The authors believe that it is not necessary for colleague dentists to only consider orthodontic therapy as an elective procedure solely for esthetic purposes. Rather it should be a front line therapeutic modality that is implemented immediately as treatment for and prevention of periodontal disease and as protective therapy from the risk of systemic disease. Whether a new patient is evaluated during a hygiene appointment or during a new patient consultation appointment, a specific protocol of cleaning or scaling and root planning of teeth should be supplemented with the Orthoeruption ${ }^{\mathrm{TM}}$ of malpositioned teeth. After diagnosis and the identification of lingually inclined teeth this new dental hygiene protocol consists of the immediate application of orthodontic brackets on the anterior lingually inclined teeth for patient comfort and ease of treatment (Figure 2) followed by brackets on other posterior lingually inclined teeth as described in our previous publications. ${ }^{29-38}$ Patients recognize the practicality of this protocol and readily accept this treatment because the root movement needed for optional positioning of teeth for professional and personal dental hygiene can take only sixty to ninety days with an additional sixty to ninety days to refine occlusion. Clinicians recognize the ease and efficiency of implementing this new protocol.

Orthodontic treatment planning is quickly evolving from both an elective procedure to correct crowding for esthetic purposes to a preventive procedure. Malocclusion including the prevalence of malpositioned and thus periodontally compromised teeth is one of the most important oral problems in the world population. There is clear understanding with scientific validation that malpositioned teeth have a unique microflora of pathogenic bacteria that causes local damage by way of periodontal disease and those individual pathogens are associated with a variety of systemic diseases. This therefore places orthodontic treatment planning or Orthoeruption ${ }^{\mathrm{TM}}$ as a key therapeutic treatment modality. The orthodontic and periodontal diagnostic combination should be common practice for the dental clinician especially with the increased interest in the orthodontic treatment of the adult patient. Even when considering modulation by the host immune response or environmental or structural risk factors, the periodontal bacterial microflora, especially in malpositioned teeth can cause localized disease which can elicit systemic effects primarily through the dissemination of proinflammatory constituents. Adding orthodontic treatment planning for periodontally involved malpositioned / lingually inclined teeth provides multiple benefits which include protection against this pathogenic microflora, improved access for professional and patient oral hygiene, protection against possible systemic disease manifestations and improvement of function and esthetics (Figure 3). Immediately moving lingually inclined teeth is part of or a continuation of cleaning these teeth.

\section{New Dental Hygiene Protocol for Systemic Disease Prevention}
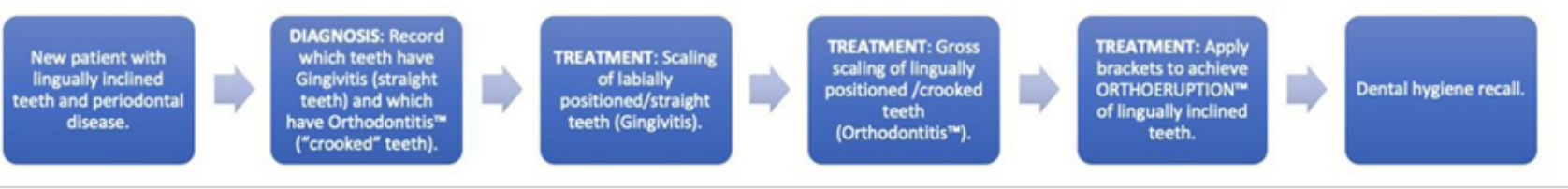

Figure $2 \mathrm{~A}$ new dental hygiene protocol which emphasizes orthoeruption ${ }^{\mathrm{TM}}$ of lingually inclined teeth.

The authors are well aware of choices patients have in selecting orthodontic systems - especially those systems that promise esthetic improvements in short periods of time irrespective of proper arch or root alignment. It is only through the patented methods of Fastbraces Technologies ${ }^{\circledR}$ that a patient can simultaneously leverage multiple benefits in a relatively short period of time to improve esthetics, naturally align arch form, upright malpositioned roots while aligning teeth for proper dental hygiene. All this while simultaneously eliminating a bacterial microflora associated with malpositioned teeth and providing protection against the possible systemic or health related effects of bacteria that originate in an unhealthy mouth. The treatment value of Fastbraces Technologies ${ }^{\circledR}$ in this relatively short time is noteworthy. Therefore, immediately integrating orthodontic treatment planning as a key therapeutic option in everyday dental hygiene treatment planning is compelling. 

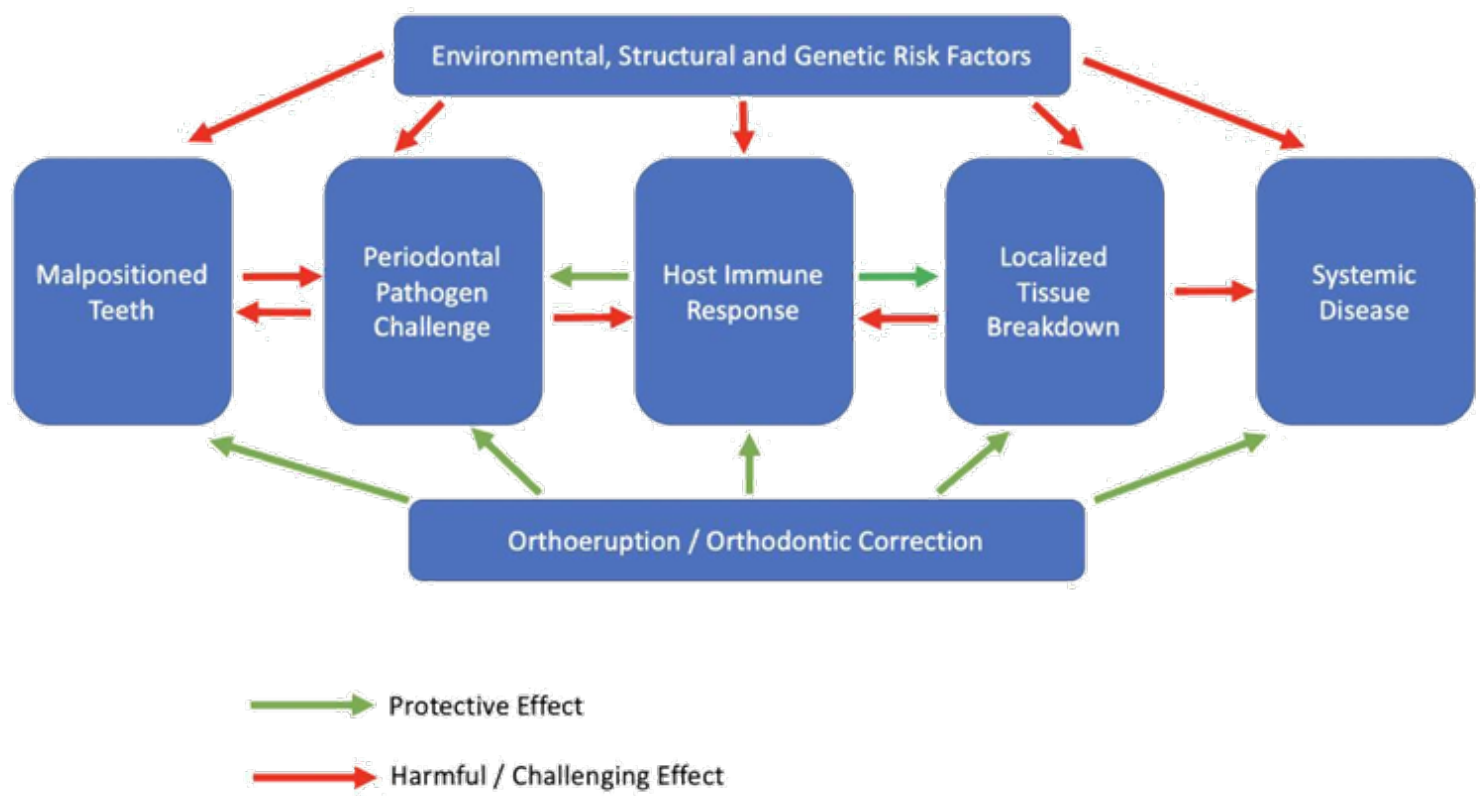

Figure 3 Pathogenesis with possible systemic manifestations of periodontal disease. Treatment planning to include orthoeruption ${ }^{\text {TM }}$ or orthodontic correction as protection against periodontal pathogenic bacteria and systemic disease.

\section{References}

1. Monsarrat P, Blaizot A, Kémoun P, et al. Clinical research activity in periodontal medicine: a systematic map- ping of trial registers. J Clin Periodontol. 2016;43(5):390-400

2. Kuehn MJ, Kesty NC. Bacterial outer membrane vesicles and the hostpathogen interaction. Genes Dev. 2005;19(22):2645-2655.

3. Bascones-Martínez A, Figuero-Ruiz E. Periodontal diseases as bacterial infection. Med Oral Patol Oral Cir Bucal. 2004;9 Suppl:92-100.

4. Byrne SJ, Dashper SG, Darby IB, et al. Progression of chronic periodontitis can be predicted by the levels of Porphyromonas gingivalis and Treponema denticola in subgingival plaque. Oral Microbiol Immunol. 2009;24(6):469-477.

5. Gemmell E, Bird PS, Carter CL, et al. Effect of Fusobacterium nucleatum on the $\mathrm{T}$ and $\mathrm{B}$ cell responses to Porphyromonas gingivalis in a mouse model. Clin Exp Immunol. 2002;128(2):238-244.

6. O’Brien-Simpson NM, Paolini RA, Reynolds EC. RgpA-Kgp peptidebased immunogens provide protection against Porphyromonas gingivalis challenge in a murine lesion model. Infect Immun . 2000;68(7):4055-4063.

7. Simonson LG, McMahon KT, Childers DW, et al. Bacterial synergy of Treponema denticola and Porphyromonas gingivalis in a multinational population. Oral Microbiol Immunol. 1992;7(2):111-112.

8. Ellen RP, Galimanas VB. Spirochetes at the forefront of periodontal infections. Periodontology 2000. 2005;38:13-32.

9. Cavrini F, Sambri V, Moter A, et al. Molecular detection of Treponema denticola and Porphyromonas gingivalis in carotid and aortic atheromatous plaques by FISH: report of two cases. Journal of Medical Microbiology. 2005;54(1):93-96.
10. Hashimoto M, Ogawa S, Asai Y, et al. Binding of Porphyromonas gingivalis fimbriae to Treponema denticola dentilisin. FEMS Microbiology Letters. 2003;226(2):267-271.

11. Chung, Vanarsdall. Comparison of microbial composition in the subgingival plaque of adult croweded versus non-crowded dental regions. Int J Adult Orthod Orthognath Surg. 2000;15(4):321-330.

12. Thornberg MJ, Riolo CS, Bayirli B, et al. Periodontal pathogenlevels in adolescents before, during, and after fixed orthodontic appliance therapy. Am J Orthod Dentofacial Orthop. 2009;13(1):95-98.

13. Raza, SS; Sultan, OW; Sohail, MR. Gram-negative bacterial endocarditis in adults: state-of-the-heart. Expert review of anti-infective therapy. 2010;8(8):879-885.

14. Cavrini F, Sambri V, Moter A, et al. Molecular detection of Treponema denticola and Porphyromonas gingivalis in carotid and aortic atheromatous plaques by FISH: report of two cases. J Med Microbiol. 2005;54(Pt 1):93-96.

15. Ishihara $\mathrm{K}$, Nabuchi $\mathrm{A}$, Ito $\mathrm{R}$, et al. Correlation between detection rates of periodontopathic bacterial DNA in coronary stenotic artery plaque [corrected] and in dental plaque samples. J Clin Microbiol. 2004;42(3):1313-1315.

16. Narikiyo M, Tanabe C, Yamada $\mathrm{Y}$, et al. Frequent and preferential infection of Treponema denticola, Streptococcus mitis, and Streptococcus anginosus in esophageal cancers. Cancer Sci. 2004;95(7):569-574.

17. Riviere GR, Riviere KH, Smith KS. Molecular and immunological evidence of oral Treponema in the human brain and their association with Alzheimer's disease. Oral Microbiol Immunol. 2002;17(2):113118

18. Fetler L, Amigorena S. Neuroscience. Brain under surveillance: The microglia patrol. Science. 2005;309(5733):392-393. 
19. Schram MT, Euser SM, de Craen AJ, et al. Systemic markers of inflammation and cognitive decline in old age. $J$ Am Geriatr Soc. 2007;55(5):708-716.

20. Arosio B, Trabattoni D, Galimberti L, et al. Interleukin-10 and interleukin-6 gene polymorphisms as risk factors for Alzheimer's disease. Neurobiol Aging. 2004;25(8):1009-1015.

21. Signat B, Roques C, Poulet $\mathrm{P}$, et al. Fusobacterium nucleatumin Periodontal Health and Disease. Curr Issues Mol Biol. 2011;13(2):25-36.

22. Han YW, Redline RW, Li M, et al. Fusobacterium nucleatum Induces Premature and Term Stillbirths in Pregnant Mice: Implication of Oral Bacteria in Preterm Birth. Infect Immun. 2004;72(4):2272-2279.

23. Yu J, Feng Q, Wong SH, et al. Metagenomic analysis of faecal microbiome as a tool towards targeted non-invasive biomarkers for colorectal cancer. Gut. 2017;66(1):70-78.

24. Rubinstein MR, Wang X, Liu W, et al. Fusobacterium nucleatum promotes colorectal carcinogenesis by modulating E-cadherin/ $\beta$-catenin signaling via its FadA adhesin. Cell Host Microbe. 2013;14(2):195-206.

25. Mima K, Nishihara R, Qian ZR, et al. Fusobacterium nucleatum in colorectal carcinoma tissue and patient prognosis. Gut. 1980;65(12):1973-1980.

26. Kostic Aleksandar D, Chun E, Robertson Lauren, et al. Fusobacterium nucleatum potentiates intestinal tumorigenesis and modulates the tumor immune microenvironment. Cell Host \& Microbe. 2013;14(2):207-215.

27. LaGier MJ, Threadgill DS. Identification of novel genes in the oral pathogen Campylobacter rectus. Oral Microbiol Immunol. 2008;23(5):406-412.

28. Arce RM, Barros SP, Wacker B, et al. Increased TLR4 expression in murine placentas after oral infection with periodontal pathogens. Placenta. 2009;30(2):156-162.
29. Viazis AD, Viazis E, Pagonis TC. The Concept of a New Dental Disease: Orthodontosis and Orthodontitis. J Dental Health Oral Disord Therapy. 2014;1(5):1-5.

30. Viazis AD, Viazis E, Pagonis TC. Orthodontic Diagnosis Based upon Alveolar Bone Morphology. J Dent Health Oral Disord Ther. 2017;8(8):00316.

31. Viazis AD, Pagonis TC. The Viazis Classification of Malocclusion. $J$ Dent Health Oral Disord Ther. 2018;9(5):363-371.

32. Viazis AD, Pagonis TC. Orthodontitis: the distinct gingivitis around malpositioned teeth. J Dent Health Oral Disord Ther. 2019;10(2):171-177.

33. Viazis AD, Viazis E, Pagonis TC. The Biology of Orthodontic Treatment Time-Days versus Years. J Dent Health Oral Disord Ther. 2017;8(1):00268.

34. Viazis AD, Viazis E, Pagonis TC. Alveolar Bone Growth with Orthoeruption. J Dent Health Oral Disord Ther. 2017;7(5):00258.

35. Viazis AD, Thaveeprungsiporn M, Pagonis TC. Alveolar Bone Remodeling and Development after Immediate Orthodontic Root Movement. J Dent Health Oral Disord Ther. 2017;6(2):00195.

36. Viazis AD, Viazis E, Pagonis TC. Non-surgical Orthodontic Adult Molar Crossbite Correction and Sleep Apnea. J Dent Health Oral Disord Ther. 2016;5(5):00168.

37. Viazis AD, Viazis E, Pagonis TC. Non-Surgical Orthodontic Treatment of an Orthognathic Surgical Case. J Dent Health Oral Disord Ther. 2016;4(5):00128.

38. Viazis AD, Viazis E, Pagonis TC. The Fallacy of Tongue Thrust and Non-Surgical Treatment of a Severe Anterior Open Bite. J Dent Health Oral Disord Ther. 2016;4(4):00120. 